\section{La salud pública en Cuba y su experiencia internacional (1959-2005)}

\section{Public health in Cuba and its international experience (1959-2005)}

Enrique Beldarraín Chaple

Profesor de Salud Pública,

Facultad de Medicina "Calixto García"

La Habana, Cuba

ebch@infomed.sld.cu
BELDARRAÍN CHAPLE, E.: La salud pública en Cuba y su experiencia internacional (1959-2005).

História, Ciências, Saúde - Manguinhos, v. 13, n. 3, p. 709-16, julio-sept. 2006.

En los últimos 42 años de existencia, el sistema de salud cubano se ha caracterizado también por una amplia experiencia y labor internacional que se inició en 1962 con el envío de la primera misión médica cubana a Argelia y desde ese momento se ha colaborado con más de 101 países de Asia, África y América Latina, desde misiones de ayuda por desastres naturales hasta la colaboración con personal médico en regiones donde antes no existían, en la elaboración de planes de desarrollo médico con algunos países según sus necesidades. También se ha desarrollado una colaboración en el terreno de la formación de los recursos humanos, con el inicio de los estudios de medicina de cientos de miles de jóvenes de todos los países de América, e incluso de algunos de Asia y África.

PALABRAS CLAVE: sistema de salud; historia; colaboración internacional; Cuba.

BELDARRAÍN, CHAPLE E.: Public health in Cuba and its international experience (1959-2005).

História, Ciências, Saúde - Manguinhos, v. 13, n. 3, p. 709-16, July-Sept. 2006.

The past 42 years of Cuba's healthcare system have been characterized, among other things, by broad-based international experience and projects, which began in 1962 with the deployment of the first Cuban medical mission to Algeria. Since then, it has collaborated with more than 101 countries in Asia, Africa and Latin America, providing aid missions to areas suffering from natural disasters and sending medical staff to regions where they did not exist before. It has also helped some countries draw up medical development plans according to their specific needs. Cooperation with human resource training and development has also been carried out, with the result that hundreds of thousands of youths have been able to start their medical studies in countries all over the Americas and in some nations from Asia and Africa.

KEYWORDS: health system; history; international cooperation; Cuba. 


\section{Desarrollo}

C uando, se prestó ayuda a Chile, en marzo de 1960, a causa del terremoto que lo afectó, comenzó a vislumbrarse una característica que será dominante en todo el desarrollo posterior del nuevo sistema de salud iniciado en 1959: la colaboración médica con otros países necesitados, tanto por motivos de accidentes o catástrofes naturales, como por déficit de profesionales médicos. En la década de los años de 1960, se inició la reforma de salud en Cuba que terminó su primera etapa en 1970 con la formación de un sistema de salud único e integral, público y de cobertura nacional.

En esta etapa se introdujeron los principios de la salud pública socialista, como rectores de la práctica de salud del gobierno. Esos principios son: la salud es un derecho de la población; la salud de la población es responsabilidad del estado; los servicios de salud alcanzan a toda la población por igual; las prácticas de salud tendrán una sólida base científica; las acciones de salud tendrán una orientación preventiva; la participación social es inherente al manejo y desarrollo de los servicios de salud; la solidaridad internacional será práctica de los servicios de salud (Rojas et al., 2000, p. 7).

Uno de estos principios ha tenido un impacto muy grande a escala internacional y es el relativo a la solidaridad internacional que se ha brindado a países necesitados de atención médica. Este servicio se inició en fecha tan temprana como 1960, cuando la República de Chile sufrió intensos terremotos. De Cuba llegó a ese país una brigada de personal médico y paramédico, así como medicamentos, práctica mantenida hasta la actualidad cada vez que ha existido una catástrofe natural. Ahí están los casos de Perú (1970), otra vez Chile (1971), Nicaragua (1972 y 1974) y los de la ayuda más reciente por el Huracán Micht en Centroamérica y las inundaciones de Venezuela.

A partir de1963 se inició la colaboración internacional sistemática con los países en vías de desarrollo que tenían necesidad de apoyo con personal médico y técnicos de la salud: Argelia y Vietnam (1963), Malí (1965), Congo Brazzaville (1966) y Guinea Conakry (1967). En la década de 1970 se extendió a África, Asia y América y se mantiene hasta el día de hoy en los tres continentes señalados.

\section{La colaboración médica internacional y el programa integral de salud}

Desde los momentos iniciales de la primera misión de este tipo que se inició con la emergencia del desastre natural sufrido por Chile en 1960 ya mencionada y el desarrollo desde entonces hasta el presente de trabajo de misiones y equipos médicos cubanos a diversos países de Asia, África y América Latina, a finales del la 
década de 1990, después de las afectaciones ocasionadas por el Huracán Mitch en Centroamérica, Cuba brindó una ayuda intensa con equipos y personal médico a los países de la región y decidió además organizar un programa integral de atención de salud que incluyera todos los aspectos de las necesidades de salud de estas poblaciones. Este programa funciona exitosamente en un grupo de países a los que actualmente ofrece colaboración médica.

Entre 1963 y 1999 participaron en los programas de colaboración 40.175 trabajadores de la salud en 83 países. En 1999 fueron 3.418 trabajadores en 57 países. (Dotres, 2001, p. 35). En noviembre del 2000, participaba en el Programa Internacional de Salud cubano el personal siguiente:

Tabla I - Personal, por país

\begin{tabular}{lcr}
\hline País & Médicos & Total \\
\hline Belice & 72 & 109 \\
Guatemala & 358 & 456 \\
Honduras & 122 & 139 \\
Haití & 231 & 451 \\
Venezuela & 71 & 107 \\
Paraguay & 49 & 49 \\
Malí & 91 & 102 \\
Gambia & 201 & 241 \\
Burkina Faso & 2 & 3 \\
Níger & 88 & 90 \\
Ghana & 105 & 141 \\
Guinea Ecuatorial & 89 & 141 \\
Zimbabwe & 97 & 103 \\
Camboya & 8 & 10 \\
\hline Total: & $\mathbf{I . 5 8 4}$ & $\mathbf{2 . 1 4 2}$ \\
\hline
\end{tabular}

Fuente: Dirección Nacional de Estadísticas, Minsap.

Paralelamente a esta presencia de personal médico cubano en otros países del tercer mundo, se diseñó en Cuba una estrategia de formación de recursos humanos en el campo de la salud dirigido a ciudadanos de esas naciones.

Desde 1966 hasta 1999 se han graduado 9.075 estudiantes extranjeros de más de 106 países. Cuba ha formado más de 1.200 profesionales fundamentalmente en la especialidad de medicina.

En 1998, a raíz de las afectaciones causadas por los huracanes George y Mitch, los gobiernos de Centroamérica y el Caribe solicitaron ayuda de la comunidad internacional. Cuba respondió de inmediato y manifestó la disposición de desarrollar un programa integral de salud con el que se beneficiaron Honduras, Guatemala, Haití, Belice, Venezuela, Paraguay, Níger, Gambia, Zimbabwe, Guinea Ecuatorial, Malí, Ghana, y Camboya. Este programa posteriormente se amplió a Chad, Guinea Bissau, Namibia, Liberia, Benin y Cabo Verde. 
Para garantizar el sostenimiento del programa en los países, se trabaja en la formación y capacitación de recursos humanos. En este contexto se creó la Escuela Latinoamericana de Ciencias Médicas, con una matrícula de 3.400 y estudiantes de 23 países, donde estaban representadas 42 etnias en el curso 1999-2000. Esta escuela es una universidad que tiene la misión de formar médicos generales básicos, orientados hacia la atención primaria de salud como escenario fundamental de su atención profesional, con una elevada preparación científica, humanista, ética y solidaria; capaces de actuar en su entorno de acuerdo con las necesidades de la región para el desarrollo humano sostenible.

El desastre provocado por los huracanes George y Mitch en 1998, que afectó profundamente a las economías de los países centroamericanos y caribeños, y que ocasionó además la pérdida irreparable de miles de vidas, tuvo la respuesta cubana de la concepción del Programa Integral de Salud, al que se integra esta escuela.

La Escuela Latinoamericana de Medicina - ELAM tiene la misión de formar gratuitamente como médicos a jóvenes de esos países, los que luego de un lapso de seis años retornarán a sus comunidades para contribuir al sostenimiento de sus sistemas de salud. Fundada y concebida como un proyecto científico-pedagógico que daba respuesta a las necesidades de profesionales de la salud en estos países, hoy acoge a estudiantes de 24 países, 19 de ellos latinoamericanos, 4 africanos y de los Estados Unidos de América, con 100 etnias (69 de latinoamérica y 31 africanas). La matrícula es de 1.500 estudiantes por año y un total de 8.600 estudiantes en los diferentes años de la carrera, distribuidos en las facultades de medicina del país.

En la actualidad estudian 10.000 alumnos y en el pasado curso académico, 2004-2005, se efectuó la primera graduación, recibiendo el título de médico casi 1.300 estudiantes.

Tabla 2 - Matrícula por países, curso 2003-2004

\begin{tabular}{lrlr}
\hline País & $\mathbf{n}^{\circ}$ & País & $\mathbf{n}^{\circ}$ \\
\hline Argentina & 283 & Guinea Bissau & 7 \\
Belice & 49 & Guinea Ecuatorial & 69 \\
Bolivia & 333 & Honduras & 581 \\
Brasil & 282 & México & 305 \\
Cabo Verde & 29 & Nicaragua & 473 \\
Chile & 266 & Nigeria & 97 \\
Costa Rica & 175 & Panamá & 316 \\
Colombia & 396 & Paraguay & 425 \\
Ecuador & 414 & Perú & 331 \\
El Salvador & 478 & Rep. Dominicana & 277 \\
Estados Unidos & 54 & Uruguay & 240 \\
Guatemala & 564 & Venezuela & 515 \\
\hline & & Total: & $\mathbf{8 . 5 9 1}$ \\
\hline
\end{tabular}

Fuente: Dirección Nacional de Estadísticas, Ministerio Salud Pública. Comunicación personal. 
En Santiago de Cuba, se inauguró en 1999 la Escuela Caribeña de Medicina, donde estudiaban 254 jóvenes haitianos y 51 malienses, en el mismo curso (Morales, 2001, p. 118). Estos altos centros docentes tienen como objetivo fundamental formar a jóvenes médicos que, una vez graduados, irán a trabajar a comunidades rurales de difícil acceso y de bajos ingresos económicos en sus respectivos países. Los alumnos se han escogido entre muchachos carentes de recursos en sus países de origen para realizar estos estudios y pertenecientes a estas comunidades de difícil acceso geográfico, a donde regresarán a brindar su atención médica una vez graduados.

En el resto de las facultades de medicina cubanas estudian más de 1.800 jóvenes de diferentes países. En sus aulas de enseñanza de especialidades de todo tipo vinculadas con la salud había, en el curso 1999-2000, una matrícula total de 4.500 estudiantes de 90 países (Morales, 2001, p. 119). Otra forma de cooperación del Programa Integrado de Salud se ha manifestado en la fundación de facultades de medicina con el apoyo de los directivos y profesores cubanos en algunos países de África, como Ghana y Guinea Ecuatorial.

En la actividad asistencial, este contingente - del Programa Integrado de Salud- había consultado hasta el año 2000 a 5.177.837 personas, muchas de las cuales nunca antes habían recibido servicios de salud. Este personal realizó 28.681 partos con asistencia especializada y en condiciones de seguridad. Las consultas a la población infantil ascendieron a la cifra de 1.135.431 niños, de ellos 173.997 menores de un año. Se realizaron 47.794 intervenciones quirúrgicas, se aplicaron dosis completas de vacunas a 178.633 personas y se habían realizado 380.453 actividades de educación para la salud (Morales, 2001, p. 119).

Es indudable que ha sido un proyecto de salud muy humanitario, con el cual se han alcanzado logros importantes, según las cifras anteriores. Además en el plano social se ha avanzado mucho en la relación entre los equipos médicos cubanos y los pobladores de los lugares donde han desarrollado su actividad profesional.

Un resumen de la colaboración médica internacional en sus primeros 40 años de existencia, nos aportan las siguientes cifras: Entre los años 1963-2003 (40 años), un total de 67.609 colaboradores en 94 países. En este período el Ministerio de Salud Pública de Cuba colaboró en la creación de nueve facultades de medicina en diferentes países y trabajó en la educación médica en 37 facultades. En el 2004 trabajaron 22.474 colaboradores en 68 países. En el año 2004, 163 profesores trabajaron en 15 países, laborando en 20 facultades de medicina. Entre los años 1966 al 2004 se graduaron en Cuba 3.914 profesionales y 4.535 técnicos de más de 100 países (Cuba, 2005, p. 109).

Un importante lugar en la colaboración médica cubana con países amigos lo ocupa Venezuela, donde el personal de salud ha 
realizado una encomiable labor llevando asistencia médica a lugares de los cerros y otros sitios intrincados donde la misma no existía, en un plan llamado Barrio Adentro, así como en la atención altamente especializada y quirúrgica que se ha ofrecido a ciudadanos de ese país en instituciones médicas cubanas en un plan coordinado con el Ministerio de Salud de dicho país. En este momento hay casi 14.000 médicos cubanos laborando en estos programas cubanovenezolanos. Y se forman recursos humanos localmente.

Un hecho de gran importancia para la colaboración médica internacional fue la fundación de una estructura médica permanente, especializada en desastres naturales, integrados por todo tipo de personal de salud, especializados y paramédicos que se le llamó Contingente 'General Henry Reeve', nombre de un soldado norteamericano que luchó en nuestra guerra de independencia en el siglo XIX. Fue creado en primera instancia para responder a las necesidades sanitarias de la población de la ciudad norteamericana de Nueva Orleáns tras el desastre del huracán Katrina, sus servicios no fueron aceptados por el gobierno norteamericano, pero el grupo formado ha tomado una estructura permanente con entrenamiento continuo en las diversas técnicas necesarias a emplearse en los desastres naturales. Tuvo sus primeras actividades con la colaboración médica prestada a Afganistán a consecuencia del gran terremoto ocurrido en el 2005. Hacia allá se envió una brigada médica multidisciplinaria que incluyó especialidades médicas, quirúrgica, así como rehabilitación y tres hospitales de campaña que posteriormente se ampliaron a cinco. El personal médico se rota cada cierto tiempo, debido a las difíciles condiciones climáticas y geográficas.

Una nueva variante de la colaboración ha sido el tratamiento quirúrgico a varios miles de pacientes, ciudadanos pobres de América Latina y el Caribe que reciben el mismo en instituciones médicas de la Isla, fundamentalmente tratamiento quirúrgico oftalmológico, programa conocido como Operación Milagro. Los pacientes beneficiados incluyen a grupos étnicos minoritarios, indígenas, que reciben esta asistencia médica gratuita en Cuba. En la actualidad se están desarrollando centros de atención oftalmológica, integrantes de esta operación, en Venezuela y Bolivia donde los médicos cubanos realizan el trabajo asistencial y además entrenan a médicos locales en dichas técnicas.

Es interesante comentar que al principio estos grupos de colaboración médica con otros países ofrecían una práctica de asistencia médica puramente científica al modo occidental, coincidiendo con el modelo médico hegemónico que existía y se promovía en el país, de la medicina altamente desarrollada y tecnificada al estilo de los patrones de la 'medicina occidental'. En los últimos tiempos esa visión se ha modificado y se brinda un sistema de atención médica comunitaria, centrado en la promoción y prevención de salud y 
con el equipo médico viviendo en la comunidad. Esto ha abierto la práctica médica a nuevos elementos culturales y de la idiosincrasia de los pueblos en los que se trabaja, sobre todo en Centroamérica, redundando en una asistencia más acorde a las necesidades, tanto biológicas como psicológicas de las comunidades en las que trabajan estos equipos de salud, y se ha involucrado a la población activamente en programas de promoción de salud.

\section{Conclusiones}

La labor internacional que ha caracterizado a la salud pública cubana en los últimos 45 años, no se ha limitado solo al plano de la asistencia médica, sino también se ha desarrollado en el escenario de la docencia, ya sea fuera o dentro de la Isla, formando recursos humanos, profesionales de la salud de otros países que no han tenido las capacidades de formarlos, por insuficiente infraestructura $\mathrm{u}$ otras razones. Primero vinieron al país estudiantes para iniciar y completar su formación, posteriormente, los profesionales cubanos ayudaron a la creación, apertura y mantenimiento de facultades y escuelas de medicina en otros países y más tarde se mantienen colaborando como profesores en muchas instituciones universitarias de formación médica en otros países del mundo.

Los profesionales médicos cubanos han trabajado en países de los tres continentes del tercer mundo en el período analizado, llevando la asistencia médica profesional a millones de personas carentes de la misma, en lugares de muy difícil acceso donde han desarrollado la medicina preventiva y han laborado en campañas

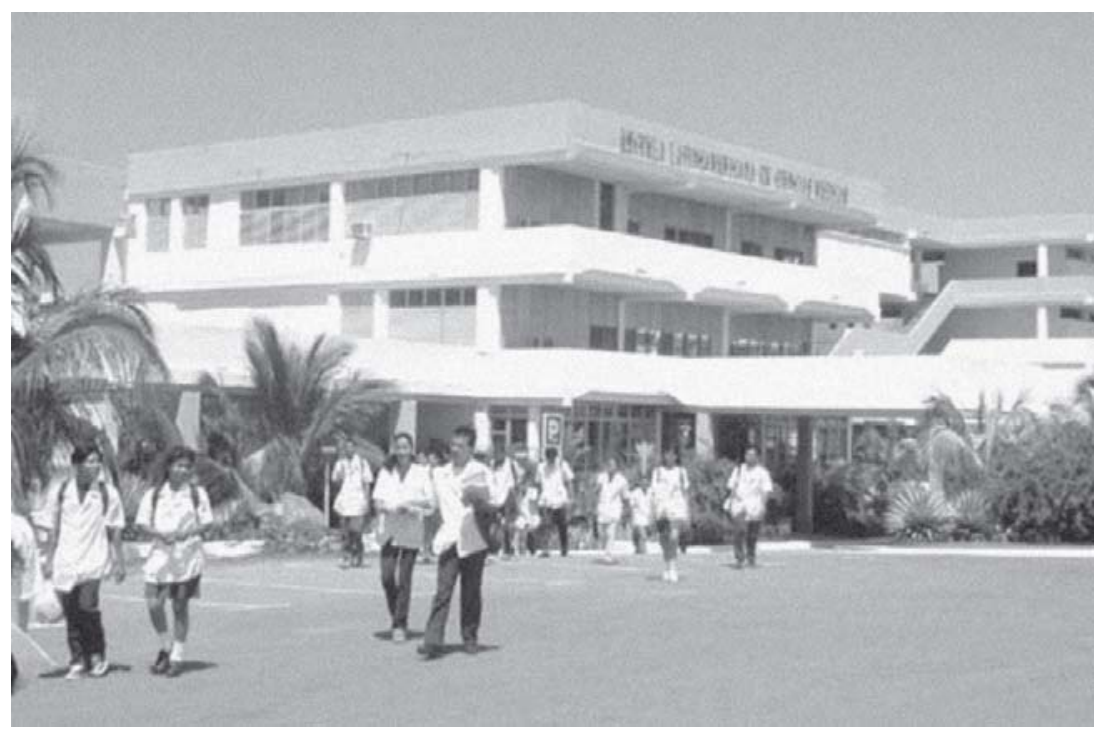

Escuela Latinoamericana de Medicina, La Habana, Cuba. 
epidemiológicas. Además han participado en peligrosas y numerosas situaciones de desastres naturales brindando su ayuda desde los primeros momentos de producidos éstos. En la actualidad se cuenta con un equipo permanente de médicos y paramédicos para trabajar en condiciones de desastres en cualquier sitio del mundo.

Se brinda como parte de esta práctica internacional, atención médica en el país a un número muy elevado de personas de América Latina que reciben atención médica fundamentalmente oftalmológica, conocido esta actividad como Operación Milagro.

\section{BIBLIOGRAFÍA}

Beldarraín, E. 2005

Cuba. Ministerio de Salud Pública

Cuba. Ministerio de Salud Pública 2004

Cuba. Ministerio de Salud Pública

Cuba. Ministerio de Salud Pública 2001

Cuba. Ministerio de Salud Pública 1999

Dotres, C. 2001

García, G. 1996

Morales, I. 2001

Rojas, F; López Serrano, E. 2000
Cambio y revolución: El surgimiento del Sistema Nacional de Salud Único en Cuba. Dynamis. Acta Hisp. Med. Sci. Hist. Illus., n. 25, p. 257-78.

Anuario estadístico 2004.

La Habana.

Anuario estadístico 2003.

La Habana.

Anuario estadístico 2002.

La Habana.

Anuario estadístico 2000.

La Habana.

La salud pública en Cuba. Hechos y cifras.

La Habana: Dirección Nacional de Estadísticas.

Sistema Nacional de Salud. Cuba, 1999. In: Memorias del Encuentro Universidad Latinoamericana y Salud de la Población. Retos y desafíos para el siglo XXI. La Habana: Minsap. p. 12-40.

La salud pública en Cuba en el período de la Revolución Socialista. In: Conferencias de Historia de la administración de salud pública en Cuba. Cuadernos de historia de la salud pública, 81, p. 131-42.

Formación de recursos humanos en salud para el Tercer Mundo. Estrategia de Cuba. In: Memorias del Encuentro Universidad Latinoamericana y Salud de la Población. Retos y desafíos para el siglo XXI. La Habana: Minsap. p. 105-20.

Revolución social y reforma sanitaria, Cuba en la década de los 60 . OPS Concursos regionales reforma sanitaria en perspectiva histórica, n. 30 . Washington (DC): OPS.

Recebido para publicación en octubre 2005.

Aprobado para publicación en mayo 2006. 